\title{
Gravity wave activity in the Martian atmosphere at altitudes 20-160 km from ACS/TGO occultation measurements
}

\author{
Ekaterina D. Starichenko ${ }^{1,2}$, Denis A. Belyaev ${ }^{1,2}$, Alexander S. Medvedev ${ }^{3}$, \\ Anna A. Fedorova ${ }^{1}$, Oleg I. Korablev ${ }^{1}$, Alexander Trokhimovskiy ${ }^{1}$, \\ Erdal Yiğit ${ }^{3,4}$, Juan Alday ${ }^{5}$, Franck Montmessin ${ }^{6}$, Paul Hartogh ${ }^{3}$, \\ ${ }^{1}$ Space Research Institute of the Russian Academy of Sciences (IKI), Moscow, Russia \\ ${ }^{2}$ Moscow Institute of Physics and Technology, Dolgoprudniy, Russia \\ ${ }^{3}$ Max Planck Institute for Solar System Research, Göttingen, Germany \\ ${ }^{4}$ Department of Physics and Astronomy, George Mason University, Fairfax, Virginia, USA \\ ${ }^{5}$ Department of Physics, University of Oxford, Oxford, UK \\ ${ }^{6}$ LATMOS/IPSL, UVSQ Université Paris-Saclay, UPMC Univ. Paris 06, CNRS, Guyancourt, France
}

\section{Key Points:}

- Observations of gravity waves from the Atmospheric Chemistry Suite instrument on board ExoMars Trace Gas Orbiter are presented

- Global distributions of the observed wave activity, potential energy, momentum fluxes and wave drag agree well with model predictions

- We found no correlation between wave amplitudes and buoyancy frequency, an extension of previously observed anticorrelation with temperature 


\begin{abstract}
The paper presents observations of gravity wave-induced temperature disturbances in the Martian atmosphere obtained with the mid-infrared (MIR) spectrometer, a channel of the Atmospheric Chemistry Suite instrument on board the Trace Gas Orbiter (ACS/TGO). Solar occultation measurements of a $\mathrm{CO}_{2}$ absorption band at $2.7 \mu \mathrm{m}$ were used for retrieving density and temperature profiles between heights of 20 and $160 \mathrm{~km}$ with vertical resolution sufficient for deriving small-scale structures associated with gravity waves. Several techniques for distinguishing disturbances from the background temperature have been explored and compared. Instantaneous temperature profiles, amplitudes of wave packets and potential energy have been determined. Horizontal momentum fluxes and associated wave drag have been estimated. The analyzed data set of 144 profiles encompasses the measurements made over the second half of Martian Year 34, from the Solar longitude $165^{\circ}$ through $355^{\circ}$. We observe enhanced gravity wave dissipation/breaking in the mesopause region of 100-130 km. Our analysis shows no direct correlation between the wave amplitude and Brunt-Väisälä frequency. It may indicate that convective instability may not be the main mechanism limiting gravity wave growth in the middle atmosphere of Mars.
\end{abstract}

\title{
Plain Language Summary
}

Gravity waves (GWs) of lower atmospheric origin continuously disturb the Martian atmosphere. While propagating upward, their amplitudes grow and eventually GWs break up or dissipate. The deposited momentum and energy are the major mechanisms driving the circulation in the thermosphere above $100 \mathrm{~km}$. Since spatial scales of GWs are relatively small, they are difficult to measure. Atmospheric Chemistry Suite (ACS) instrument on board the ExoMars Trace Gas Orbiter allows for extracting altitude profiles of density and temperature from the troposphere to the thermosphere $(20-160 \mathrm{~km})$ with high vertical resolution, around $2 \mathrm{~km}$. The instrument measures the solar spectrum occulted by the atmosphere with the carbon dioxide absorption in the middle infrared wavelength range. The observations provide latitudinal and seasonal coverage of the GW activity and its characterization on Mars. Our results allow for the first observational validation of model predictions, quantifying dynamical effects of GWs and constraining Martian general circulation models.

\section{Introduction}

The structure and circulation of planetary atmospheres are strongly affected by gravity waves (GWs), which are ubiquitous in any convectively stable atmosphere. They are primarily responsible for energy and momentum transfer from the lower to the upper atmosphere. Historically, GW-induced coupling was extensively studied in Earth's atmosphere (e.g., see reviews by Fritts \& Alexander, 2003; Yiğit \& Medvedev, 2015). With the progress in space exploration, the atmosphere of Mars has become the second beststudied example. Numerous space missions accompanied by numerical modeling have delivered ample evidence for the importance of GWs on Mars. Some of the Martian GW effects, their commonality and specifics with those on Earth, have been summarized in the recent review by Medvedev and Yiğit (2019). Observational knowledge of GW activity on Mars is crucial but still insufficient for quantifying their effects and constraining Martian general circulation models (MGCMs). Our paper addresses this problem by utilizing high-resolution occultation data obtained from the Atmospheric Chemistry Suite (ACS) instrument on board the Trace Gas Orbiter (TGO).

Observations of the Martian GWs have been conducted from orbiters by different remote sensing techniques and in situ methods. In situ measurements of GW-induced density fluctuations in the thermosphere were performed with accelerometers during aerobraking operations by several spacecraft including Mars Global Surveyor (MGS), Mars 
Odyssey (ODY), Mars Reconnaissance Orbiter (MRO), Mars Atmosphere and Volatile EvolutioN (MAVEN) and Trace Gas Orbiter (TGO) (Keating et al., 1998; Creasey et al., 2006a; Fritts et al., 2006; R. H. Tolson et al., 2005; R. Tolson et al., 2008; Withers, 2006; Jesch et al., 2019; Vals et al., 2019; Siddle et al., 2020). GWs in the upper thermosphere were also measured by Neutral Gas and Ion Mass Spectrometer (NGIMS) on board MAVEN (Yiğit et al., 2015; England et al., 2017; Terada et al., 2017). Temperature and density disturbances associated with GWs have been remotely retrieved from stellar, solar and radio occultation data as well as from limb observations (Hinson et al., 1999; Creasey et al., 2006b; Ando et al., 2012; Wright, 2012; Heavens et al., 2020; Nakagawa et al., 2020).

The shortcoming of many previous GW observation techniques is their limited altitude coverage. For example, in situ measurements were confined to a relatively narrow vertical range in the thermosphere, while radio occultation and infrared limb sounding allowed for studying the lowermost $(0-40 \mathrm{~km})$ part of the atmosphere. Remote sensing in UV permitted the extension of the altitude coverage. The Spectroscopy for the Investigation of the Characteristics of the Atmosphere of Mars (SPICAM) instrument on board Mars Express (MEX) measured temperature and density profiles between 60 and $130 \mathrm{~km}$ (Forget et al., 2009). The Imaging Ultraviolet Spectrograph (IUVS) on board MAVEN explored the thermospheric layers (100-150 km) (Medvedev et al., 2016; Gröller et al., 2018). Recently, Nakagawa et al. (2020) obtained temperature profiles from IUVS data spanning the atmosphere from 20 to $140 \mathrm{~km}$ with a vertical sampling better than $6 \mathrm{~km}$. ACS/TGO is the first IR instrument that allows for measuring in the solar occultation mode temperature and density distributions within an even broader range of altitudes (20-160 km). Since April 2018, the Middle InfraRed (MIR) spectrometer, one of the three spectrometers of ACS, delivered several hundreds of profiles, some of which having vertical resolution allowing for the study of GWs.

Smaller-scale GW-induced temperature perturbations have to be first separated from the background temperature field associated with the large-scale variations. Although this procedure has been frequently performed in the terrestrial and Martian context, there is no universal technique that can be applied to vertical profiles obtained from different instruments (Ehard et al., 2015). In this work, we explore the sensitivity of several methods for extracting GWs from the ACS MIR data and describe their applications for deriving various characteristics of the GW field (amplitudes, wave potential energy, momentum fluxes and wave drag) from the first available set of ACS/TGO data.

The paper is structured as follows. The ACS/TGO experiment and the instrument itself are outlined in Section 2. Section 3 describes the methods used in this study. In particular, retrievals of temperature profiles from measured spectra are presented in section 3.1, the techniques for extracting wave disturbances are given in section 3.2. Subsection 3.3 describes the derivation of wave activity (amplitude of wave packets) and potential energy, and 3.4 outlines the calculation of the absolute vertical flux of horizontal momentum and momentum forcing of the mean flow. The results are presented in section 4 . They include a case study (4.1), the spatial distribution of wave characteristics (4.2), and the relationship between wave amplitudes and the Brunt-Väisälä frequency (4.3). Conclusions are given in section 5 .

\section{Atmospheric Chemistry Suite Instrument on Board Trace Gas Or- biter}

ACS is a set of three infrared spectrometers for ExoMars 2016 TGO mission. It has been operating in the Martian orbit since April 2018. ACS consists of the near-(NIR), middle-(MIR) and thermal-infrared (TIRVIM) channels, that altogether cover the broad spectral range of $0.7-17 \mu \mathrm{m}$. (Korablev et al., 2018). In this paper, we use the data retrieved from the cross-dispersion echelle MIR spectrometer working in the solar occul- 
A

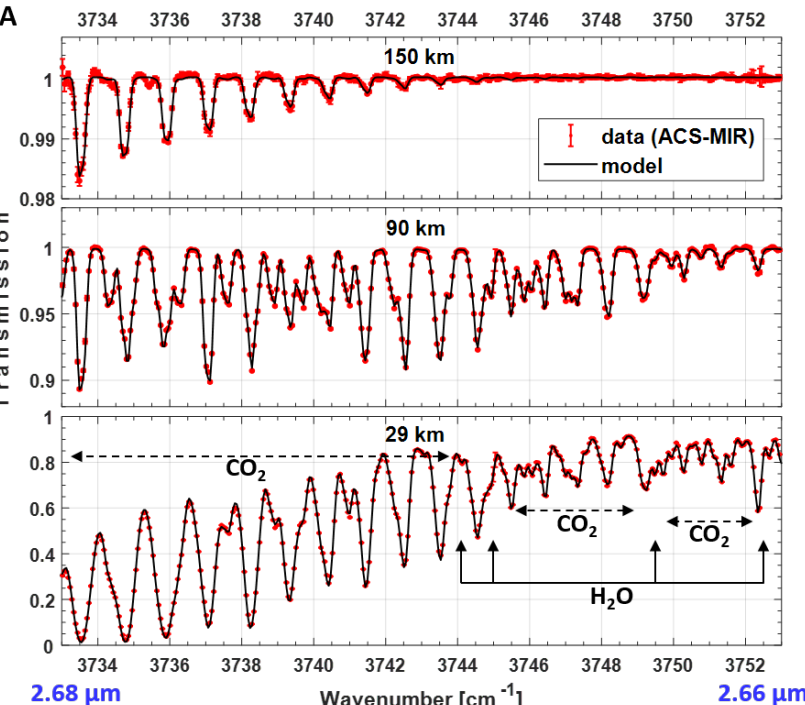

tation mode in the 2.3-4.2 $\mu \mathrm{m}$ range. This spectral coverage is achieved with a secondary dispersion grating, which can be rotated to one of 12 positions. During an occultation, the instrument is pointed to the Sun. Each measurement consists of an image at the $640 \times$ 512 pixels focal plane array (FPA), which accommodates up to 20 diffraction orders dispersed over FPA by the secondary grating. One occultation covers $0.15-0.3 \mu \mathrm{m}$ range. The instrument's resolving power is $\lambda / \Delta \lambda \sim 25000$ and the signal-to-noise ratio varies between 1000 and 10000. The vertical resolution of MIR depends on the integration time ( $\sim 2 \mathrm{~s}$ per image) and ranges from 0.5 to $2.5 \mathrm{~km}$. The transmission is obtained by division of the solar spectrum passed through the atmosphere to the reference one, which is measured above the altitude of $200 \mathrm{~km}$, where the absorption by the atmosphere is negligible.

In this study, we use the $2.66-2.68 \mu \mathrm{m}$ portion of the spectrum from the grating position \#4, the echelle diffraction order \#223, which includes a wing of the $2.7 \mu \mathrm{m} \mathrm{CO}_{2}$ absorption band (Figure 1a). Strong absorption lines of $\mathrm{CO}_{2}$ allow for retrieving tem-

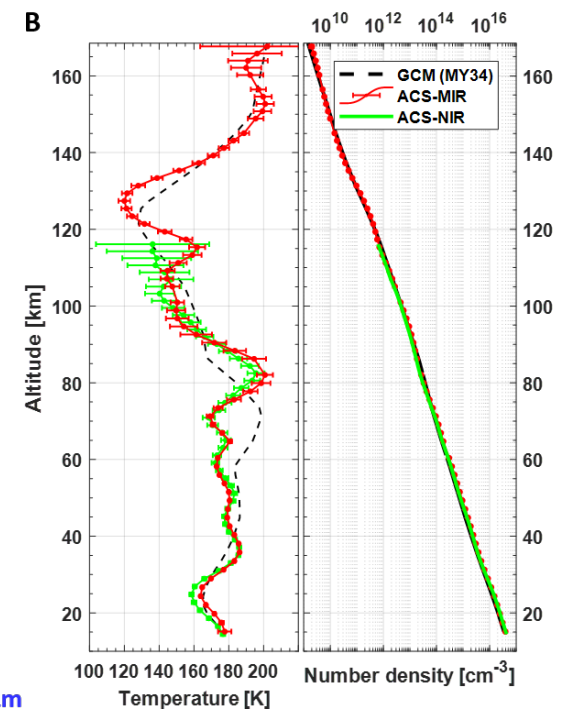

Figure 1. Spectroscopy of $\mathrm{CO}_{2}$ and $\mathrm{H}_{2} \mathrm{O}$ absorption in the diffraction order \#223 of ACSMIR (panel A) and an example of retrieved atmospheric temperature and density vertical profiles (panel B). a) Transmission spectra measured at tangent altitudes of 150, 90 and $29 \mathrm{~km}$ (red dots) on a background of the best-fitted models (black solid lines); b) Vertical profiles of temperature (left) and atmospheric number density (right) derived from the MCD (black dashed line), from ACS-MIR (red dots), and from ACS-NIR (Fedorova et al., 2020) (green dots). Error bars for the temperature values express $1-\sigma$ uncertainties of the retrievals.

perature and density in the Martian atmosphere with good sensitivity.

\section{Methods}

\subsection{Retrieval of Temperature Profiles}

The retrieval scheme consists of several iterations. On the first step, we retrieve temperature and pressure from the rotational structure of $\mathrm{CO}_{2}$ absorption bands in spectral intervals without $\mathrm{H}_{2} \mathrm{O}$ lines (see Figure 1a). A priori altitude profiles of $T(z)$ and $p(z)$ as well as one of the $\mathrm{CO}_{2}$ VMR, are taken from the Mars Climate Database (MCD) 

where the bar denotes an appropriate averaging. Generally, it implies averaging over wave phases, or spatial and temporal scales that are larger than the periods and wavelengths of contributing GW harmonics. In the case of almost instantaneous (with respect to the periods of GWs) occultation profiles, only separation in vertical scales is possible.

John and Kumar (2013) and Ehard et al. (2015) reviewed several common methods of the partition of measured temperature and/or density profiles into the "mean" and wave components. They work well if a clear separation in vertical wavelengths does exist between GWs and large-scale motions belonging to the background. This is not always the case in the Martian atmosphere, because vertical scales of disturbances associated with tides, planetary waves, and other motions may overlap with those due to GWs. It is desirable to retain the former in the background, but one still has to set a vertical scale $\Lambda_{z}$ that separates GWs from the larger-scale features. In the following, we assumed $\Lambda_{z}=30 \mathrm{~km}$. This value may lead to an overestimation of the retrieved wave activity by including non-GW perturbations, but at least no large-scale GW components are missed. Concerning the short-wavelength part of the spectrum, the limited vertical resolution favors detection of larger-scale waves, leaving out harmonics with smaller scales unobserved. Alexander (1998) has quantified this "observational filter" and pointed out that some large-scale harmonics refracted by the mean wind beyond the lowest resolution may be missing in observations as well.

We explore three methods: spectral filtering, sliding least-square polynomial fit and high-order polynomial fit. The former two have been discussed in relation to lidar and space-based measurements in the atmosphere of Earth (John \& Kumar, 2013; Ehard et al., 2015, and the references therein), while the latter was applied to profiles obtained in the terrestrial (e.g., Spiga et al., 2008) and Martian atmosphere (Yiğit et al., 2015; Terada et al., 2017; Jesch et al., 2019). Since the ACS data are distributed irregularly over the altitude, they were first interpolated (oversampled) to an evenly spaced 500-m grid. We used only the temperature data with errors $\leq 10 \mathrm{~K}$. Spectral filtering was performed using Fourier decomposition within sliding $60-\mathrm{km}$ intervals $( \pm 30 \mathrm{~km}$ around each point), and zero-order Fourier coefficients were used to calculate the background temperature. The examples are shown in Figure 2 for two characteristic profiles T(z). They visibly differ: the profile in Figure 2a (orbit 2892n1) contains large-scale disturbances, while the one in Figure 2c (orbit 3251n1) comprises mostly smaller-scale fluctuations. This method yields smooth mean temperature profiles and, as a result, large deviations from the mean (Figure $2 \mathrm{~b}$ and $\mathrm{d}$ ). This is in particular obvious below $60 \mathrm{~km}$ and in the upper part of the domain (panels $b$ and $d$ ).

For the sliding polynomial fit, we used a procedure described in the work of Whiteway and Carswell (1995). The background profiles are obtained by fitting cubic polynomials within the $60-\mathrm{km}$ sliding intervals. Observational errors were used as weights, that assign a significance to the measurements at each altitude. At first, the intervals were shifted up from the bottom to top by a certain distance (shown in Figure 2a and c for 2 and $11 \mathrm{~km}$ ), and then the procedure was repeated for the downward shifts starting from the top. The overlapping values of fits from each range were then averaged. Thus obtained profiles were then smoothed using a moving average. At the bottom of the profiles, we had to decrease the width of the sliding windows due to large spurious variations in fitted polynomials and in order to make most of the observational data. The upper and lower $4 \mathrm{~km}$ of thus obtained profiles have to be excluded anyway, because of the poor behavior of fitting polynomials, which cannot be averaged with counterparts from other sliding windows. This method occasionally produces disturbances oscillating not around zero. To correct for these numerical biases, we perform detrending by applying the Theil-Sen estimator (Theil, 1950; Sen, 1968) and fitting a linear function to the perturbation profile. The Theil-Sen estimator is a robust method, which is used for determining the linear regression taking the median of the slopes of all lines that can be drawn 
through the given dataset. The linear function is then subtracted from the profile to obtain the corrected temperature.
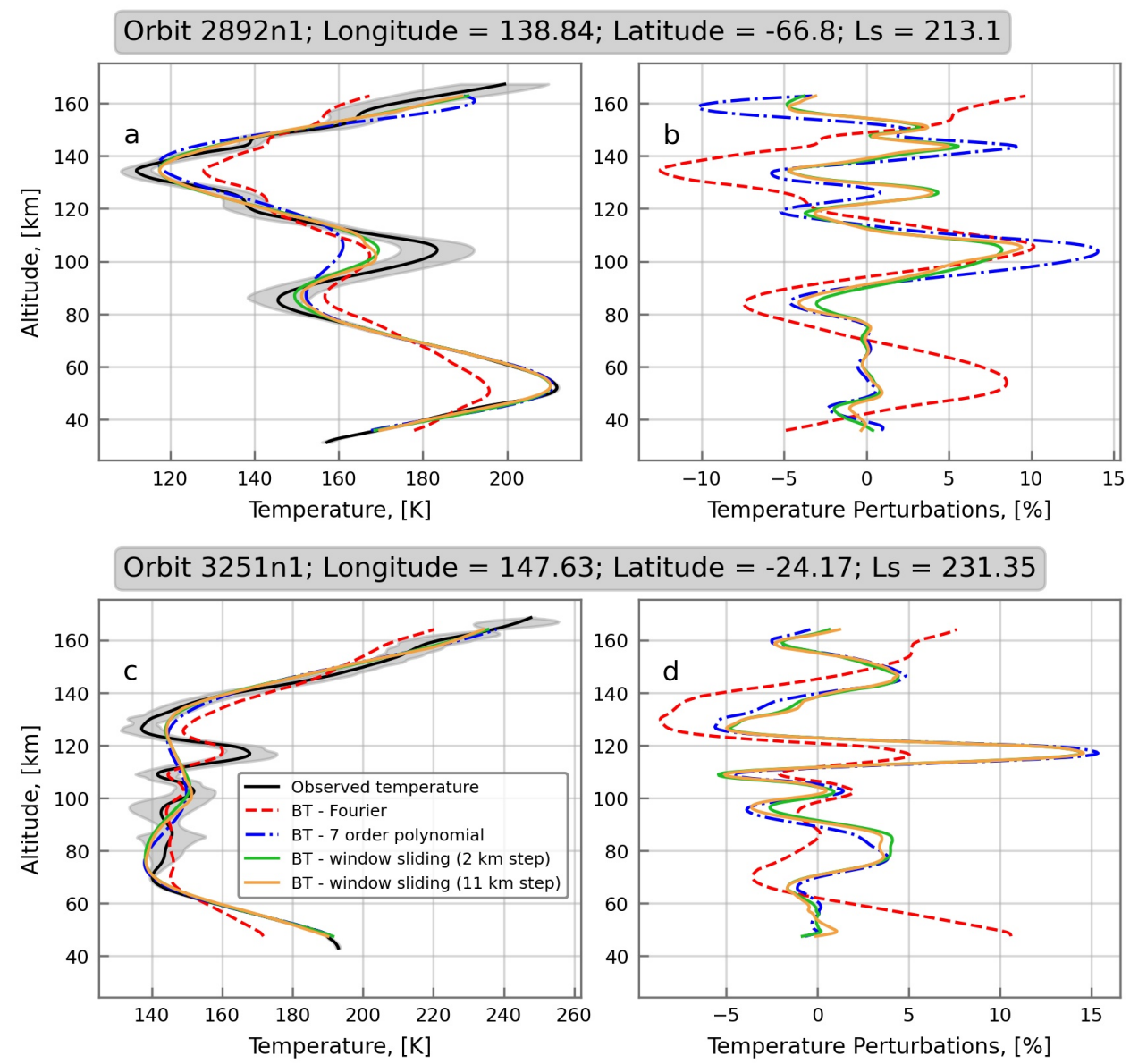

Figure 2. Separation of the observed temperature into the mean and wave components for two characteristic profiles: dominated by large vertical-scale (orbit 2892n1, upper row) and smallscale disturbances (orbit $3251 \mathrm{n} 1$, lower row). Left column is for the mean temperature $\bar{T}(z)$, the right one is for the relative perturbations $T^{\prime}(z) / \bar{T}(z)$ (in percent). The legend describes the applied methods. Red dashed lines correspond to the Fourier decomposition, green and yellow lines are for the sliding polynomial fit with 2-km and 11-km shift steps, correspondingly, and the blue lines are for the 7-th order polynomial fit. The observed temperature profiles are given with the solid black lines. Shaded area denotes the uncertainty of the measurements.

The results for the sliding polynomial fit are plotted in Figure 2 for the 2 and 11 $\mathrm{km}$ shift steps with green and yellow lines, correspondingly. It is seen that they are very close and, thus, the background and disturbances depend on the sliding step to a minor degree. The method shows some useful features in comparison with spectral filtering. The fitted mean curves in the regions of large-scale disturbances (Case 1) follow the observed temperature profiles closer (Figure 2a) and are smoother where small-scale structure dominates (Case 2) (Figure 2c, between 70 and $130 \mathrm{~km}$ ). This produces smaller wave amplitudes in Case 1, and reveals more wavy structures in Case 2. Especially plausible results are in the bottom of the profiles, where GWs are expected to have smaller amplitudes (due to larger density). 
We next explored the technique of fitting higher-order polynomials in the entire interval of heights. In particular, the seventh-order polynomial fit, which was previously used for extracting GWs on Mars (Yiğit et al., 2015; Jesch et al., 2019), produces most plausible results. They are presented in Figure 2 with dashed and dotted blue lines. It is immediately seen that thus obtained wave disturbances are in a very good agreement with those derived by the sliding polynomial fit method, especially for profiles containing small-scale features (Figure 2d). For profiles dominated by large-scale perturbations, the agreement is also good in terms of the determined vertical structure of the wave, although the magnitudes are often exaggerated (Figure $2 \mathrm{~b}$ ). The weak point of the method is that it occasionally produces spurious disturbances near the edges of the vertical domain with vertical gradients of the mean temperature directed opposite to the measured profiles. After careful consideration of the three methods applied to the available measurements, we selected the sliding third-order polynomial fit as the most appropriate and robust.

\subsection{Wave Activity and Potential Energy}

The GW field is often characterized by the magnitude of fluctuations $\left|T^{\prime}\right|=\left(\overline{T^{\prime 2}}\right)^{1 / 2}$ and wave potential energy (per unit mass)

$$
E_{p}=\frac{1}{2}\left(\frac{g}{N}\right)^{2} \overline{\left(\frac{T^{\prime}}{\bar{T}}\right)^{2}}
$$

where $N$ is the Brunt-Väisäla frequency

$$
N=\sqrt{\frac{g}{\bar{T}}\left(\frac{d \bar{T}}{d z}+\frac{g}{c_{p}}\right)}
$$

$g$ is the acceleration of gravity and $c_{p}$ is the specific heat capacity at constant pressure. The amplitude of the wave packet at a given height $\left|T^{\prime}(z)\right|$ (hereafter called "wave activity") represents an envelope of the measured profile $T^{\prime}(z)$. We calculated it by performing Fourier decomposition in each sliding $60-\mathrm{km}$ vertical interval and, based on Parceval's identity, summing up contributions of all harmonics. Examples of thus obtained envelopes and potential energy for the same selected profiles as in section 3.2 are presented in Figure 3. Blue and red dashed lines denote the quantities calculated from the entire spectrum and by accounting for contributions of only two largest harmonics. It is seen that the neglect of shorter-scale harmonics, as was occasionally done in analyses of satellite observations (e.g., Ern et al., 2004), introduces little error to the estimated GW activity. However, the neglect of short-scale harmonics may lead to a noticeable underestimation of wave potential energy, (cf. Figures $3 \mathrm{~b}$ and d).

\subsection{Momentum Flux and Momentum Deposition}

Another useful characteristic of the GW field is the vertical flux of horizontal momentum, or "momentum flux" for brevity, $\mathbf{F}=\left(F_{x}, F_{y}, 0\right)=\rho_{0}\left(\overline{u^{\prime} w^{\prime}}, \overline{v^{\prime} w^{\prime}}, 0\right)$, where $\rho_{0}$ is the mean density and $\left(u^{\prime}, v^{\prime}, w^{\prime}\right)$ are the components of wave-induced perturbations of wind velocity $\mathbf{u}^{\prime}$ along with the two horizontal and the vertical axis, correspondingly. Momentum flux is constant for conservatively propagating waves. Breaking/dissipating GWs deposit their momentum to the mean flow, thus inducing an acceleration or deceleration (depending on the sign) of the horizontal flow

$$
\left(a_{x}, a_{y}\right)=-\frac{1}{\rho_{0}} \frac{d \mathbf{F}}{d z} .
$$

The direction of the flux cannot be determined from the occultation measurements, however total (or absolute) momentum fluxes for a harmonic $F_{k, m}=\sqrt{F_{x, k, m}^{2}+F_{y, k, m}^{2}}$ can 

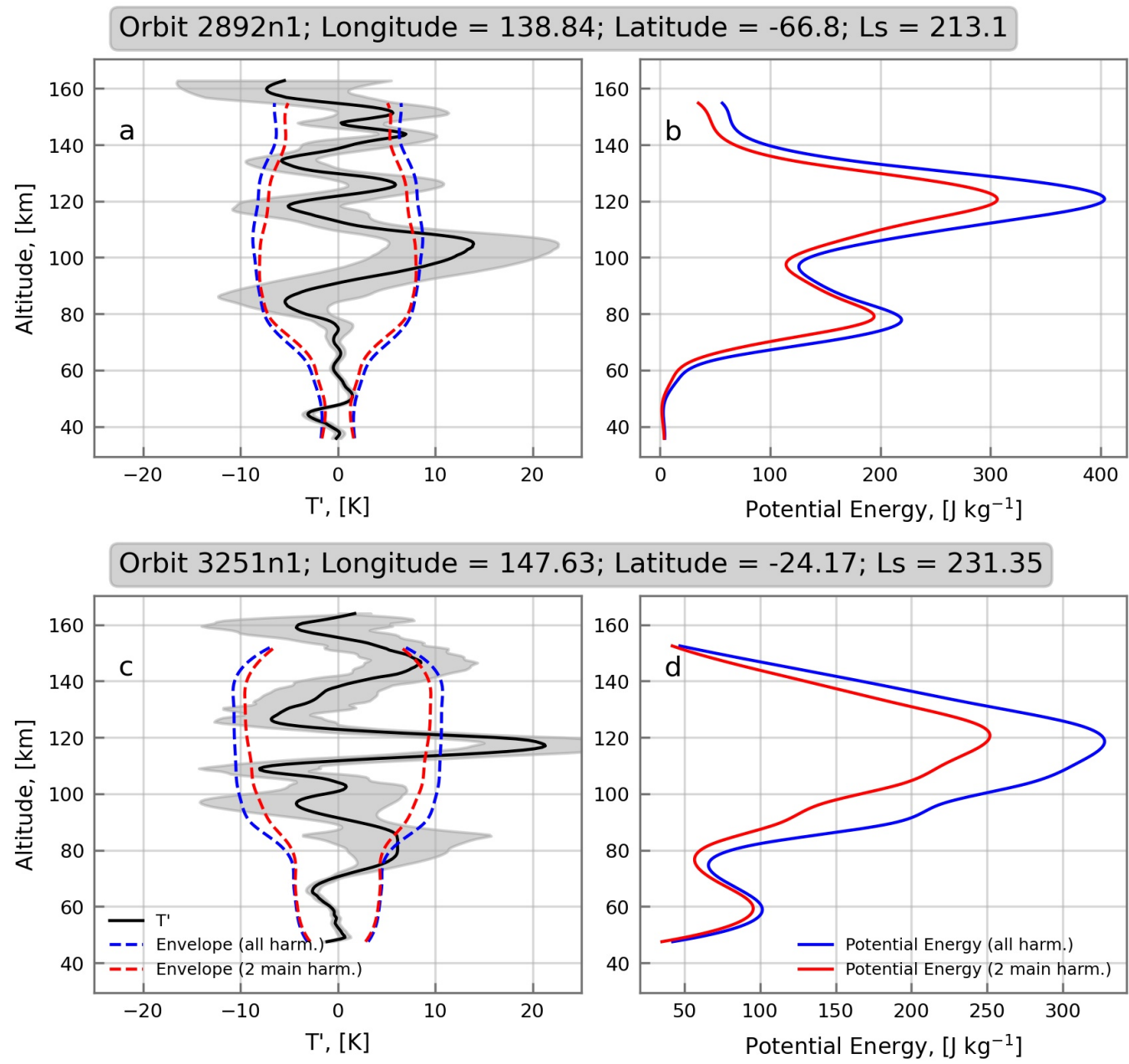

Figure 3. Wave activity $\left|T^{\prime}\right|$ (left column) and potential energy (per unit mass, right column) for the same as in Figure 2 representative profiles. Dashed blue lines indicate quantities calculated for the entire spectrum, dashed red lines are for accounting two longest harmonics only. Shaded areas denote observational errors.

be estimated (e.g., Ern et al., 2004, sect. 4):

$$
F_{k, m}=\frac{1}{2} \rho_{0} \frac{k_{h}}{m}\left(\frac{g}{N}\right)^{2}\left(\frac{\left|T_{k, m}^{\prime}\right|}{\bar{T}}\right)^{2},
$$

where $k_{h}$ and $m$ are the horizontal and vertical wavenumbers, correspondingly, and $\left|T_{k, m}^{\prime}\right|$ is the amplitude. The latter two are found from the Fourier decomposition, whereas $k_{h}$ cannot be derived from our measurements.

The total flux $F$ is the sum of contributions of individual harmonics $F=\sum_{m} F_{k, m}$. Since the horizontal wavenumber $k_{h}$ cannot be obtained from the measurements, it, therefore, serves as a scaling factor for the derived profiles of $F$ and momentum forcing (5). The densest atmospheric footprint at a target point in occultation geometry is 400-500 $\mathrm{km}$ horizontally, depending on the height. This constrains the upper limit for unresolved wavelengths. In our calculations, we assumed a representative horizontal wavelength $\lambda_{h}=$ $2 \pi / k_{h}=300 \mathrm{~km}$, the value typically used in numerical general circulation models (Yiğit 
et al., 2018), and allowing for more direct comparison with simulations. The results for two representative profiles, same as in Figures 2 and 3, are given in Figure 4. To demon-
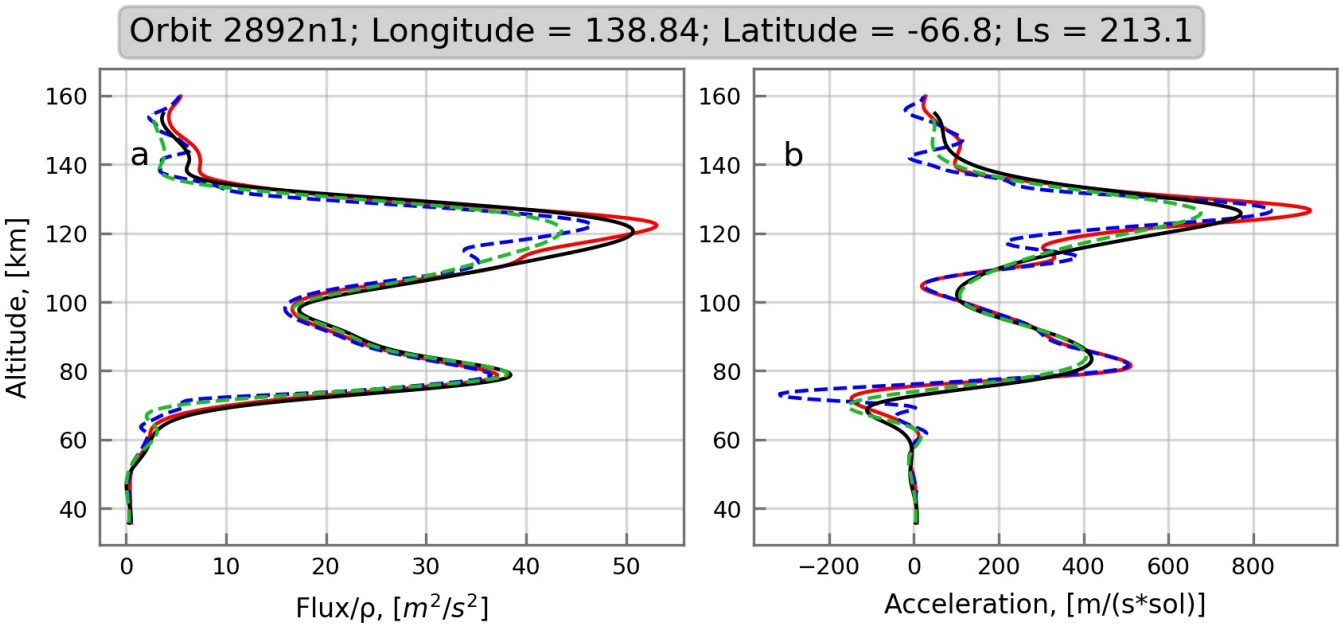

Orbit 3251n1; Longitude $=147.63 ;$ Latitude $=-24.17 ;$ Ls $=231.35$
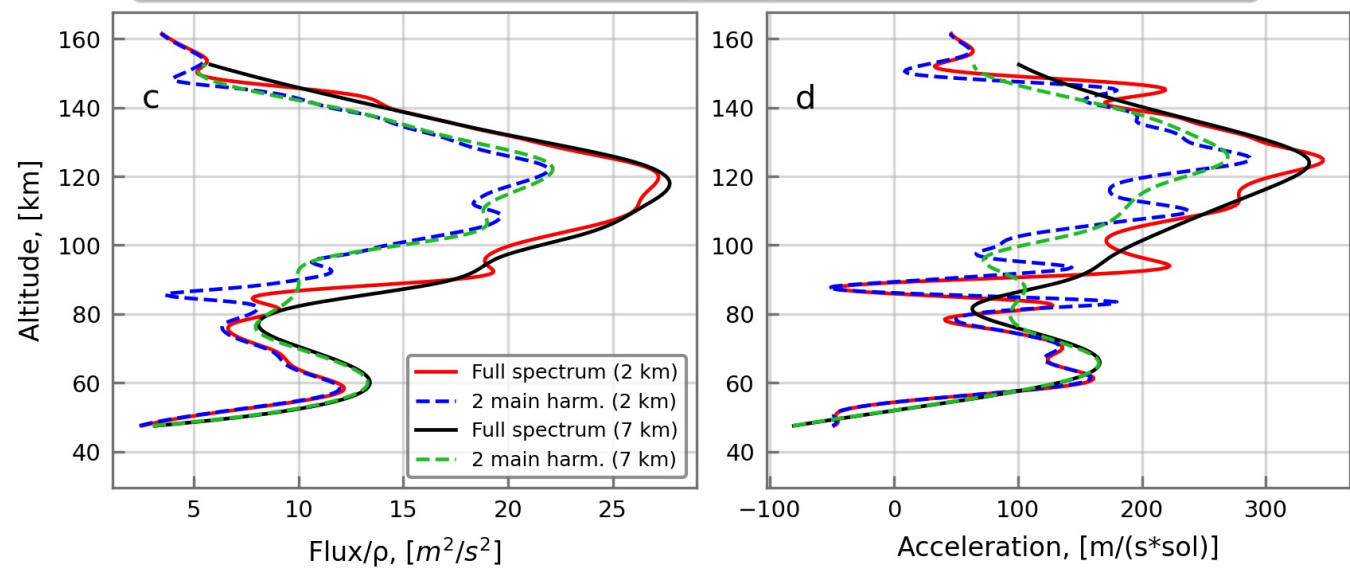

Figure 4. Absolute momentum flux (per unit mass) and the momentum forcing for two representative profiles (orbits 2892n1 and 3251n1, upper and lower rows, correspondingly). The legend describes the profiles calculated using the full spectrum and only two major harmonics along with sliding interval steps 2 and $7 \mathrm{~km}$.

strate the sensitivity of the calculations to the used parameters of the technique, we plotted with different colors the profiles of momentum fluxes (per unit mass) $F / \rho_{0}$ and GW momentum deposition, i.e., wave drag $a$ obtained from the full spectrum and taking account of only two major harmonics. In addition, the results are shown for the interval shifts 2 and $7 \mathrm{~km}$. It is immediately seen that these details play little role, and the calculations of fluxes and wave drag are very robust when the measured temperature profile is dominated by large-scale features (Figure 4, the upper row). It is different for profiles containing smaller vertical-scale disturbances (Figure 4, the lower row): their neglect leads to an underestimation of the fluxes and wave drag, and the smaller vertical shifts reveal finer structure associated with dissipation of individual spectral harmonics. 


\section{Results and Discussions}

\subsection{Case Study}

Spectral analysis of the obtained set of profiles (described in the next subsection) has demonstrated greater contribution of larger-scale disturbances in all cases. However, each individual profile was unique. Two examples with and without small vertical-scale components have been presented above. We next consider a case with a relatively broad spectrum of wave-like perturbations with large amplitudes (about twice as large as those in orbit 3251n1). The retrieved temperature for the orbit 4926n1 along with the fitted background profile are plotted in Figure 5a. The envelope in Figure 5b clearly shows that the amplitude gradually ceases its exponential growth with height and becomes nearly constant above $\sim 110 \mathrm{~km}$. The reason for this so-called wave "saturation" can be seen

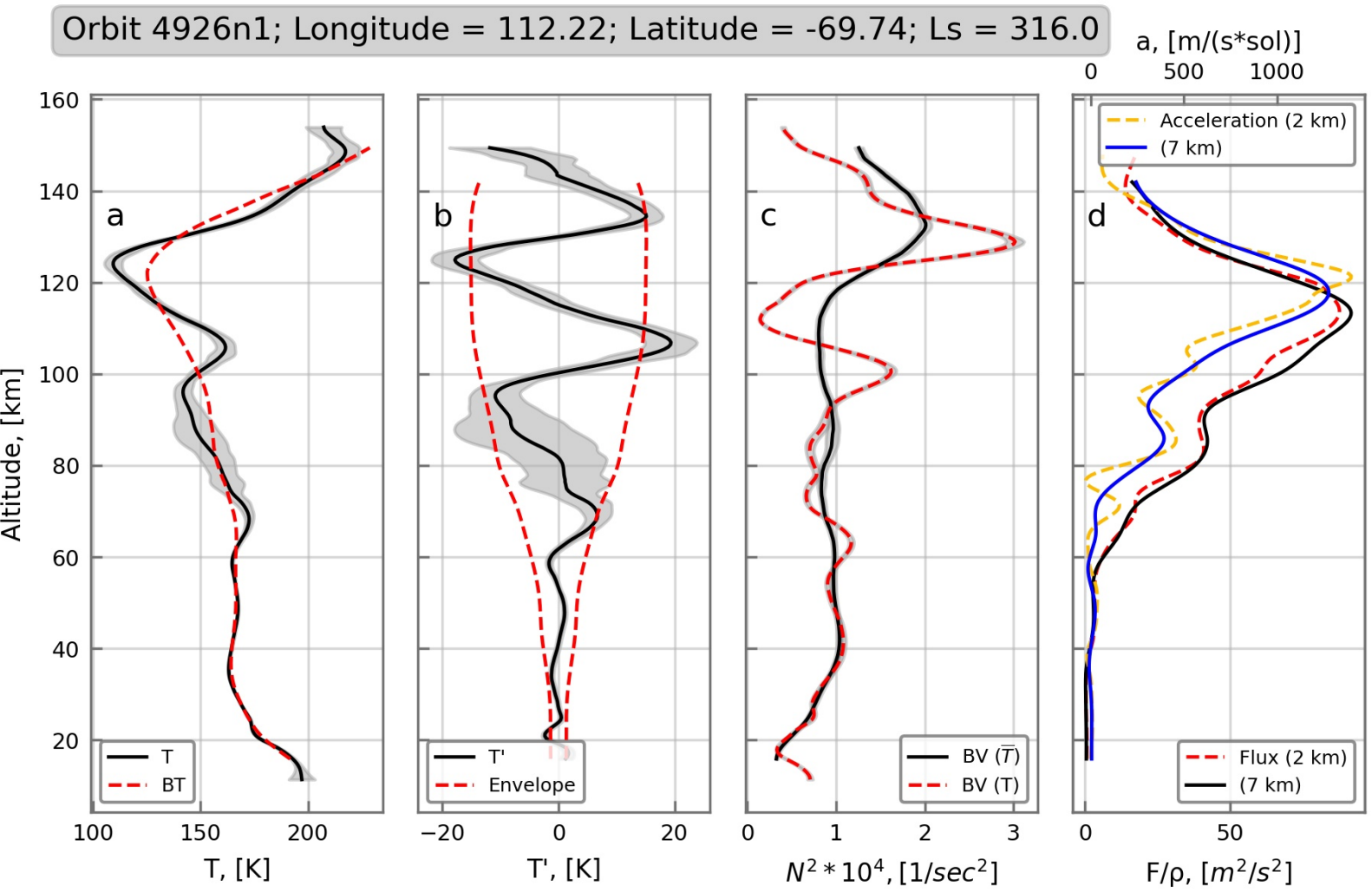

Figure 5. Vertical profiles for the orbit 4926n1. a) The measured (solid black) and fitted mean temperature (red dashed); b) wave temperature disturbance (solid black) and envelope (red dashed); c) Brunt-Väisälä frequency calculated for the mean (black) and net temperature (red dashed); d) momentum flux calculated using 2- and 7-km sliding window shifts (bottom axis, red dashed and solid black lines, correspondingly, and mean flow acceleration ("wave drag", upper axis, yellow dashed and solid blue for the 2- and 7-km steps, respectively). Shading denote observational uncertainties.

from the behavior of the squared Brunt-Väisälä frequency $N^{2}(z)$ (Figure $5 \mathrm{c}$, black). $N^{2}$ calculated from the background profiles (Figure 5c) remains relatively constant with height (up to about $120 \mathrm{~km}$ ) suggesting convective stability of the mean state. $N^{2}$ from the original profiles (see Figure 5c, red-dashed) shows large swings associated with temperature 
disturbances. Near $110 \mathrm{~km}, N^{2}$ drops almost to zero as the result of the temperature gradient (associated with a large amplitude of the disturbances) approaching the adiabatic lapse rate. Enhanced wave dissipation due to a combination of physical processes (Yigit et al., 2018) in the vicinity of the convective instability severely limits the GW amplitude, leading to the decrease of the momentum flux above this altitude and peaking of the mean flow acceleration (Figure $5 \mathrm{~d}$ ) at almost $2000 \mathrm{~m} \mathrm{~s}^{-1} \mathrm{sol}^{-1}$. In the analyzed data set, such large numbers are not common and occur only occasionally. Application of a smaller vertical shift of sliding intervals shows finer structure of the GW momentum flux and drag, but do not significantly modify the magnitudes.

\subsection{Spatial Distribution of Gravity Wave Activity}

In this section, we use the data obtained by the ACS instrument in MY34, at solar longitudes from $L_{s}=164^{\circ}$ to $354^{\circ}$. The data set contains altogether 144 occultation profiles: 84 in the northern hemisphere and 60 in the southern one. The latitudesolar longitude coverage is shown in Figure 6 with red and blue dots representing morning and evening occultation measurements, correspondingly. The longitudinal orbit coverage was fairly uniform, and is not discussed here.

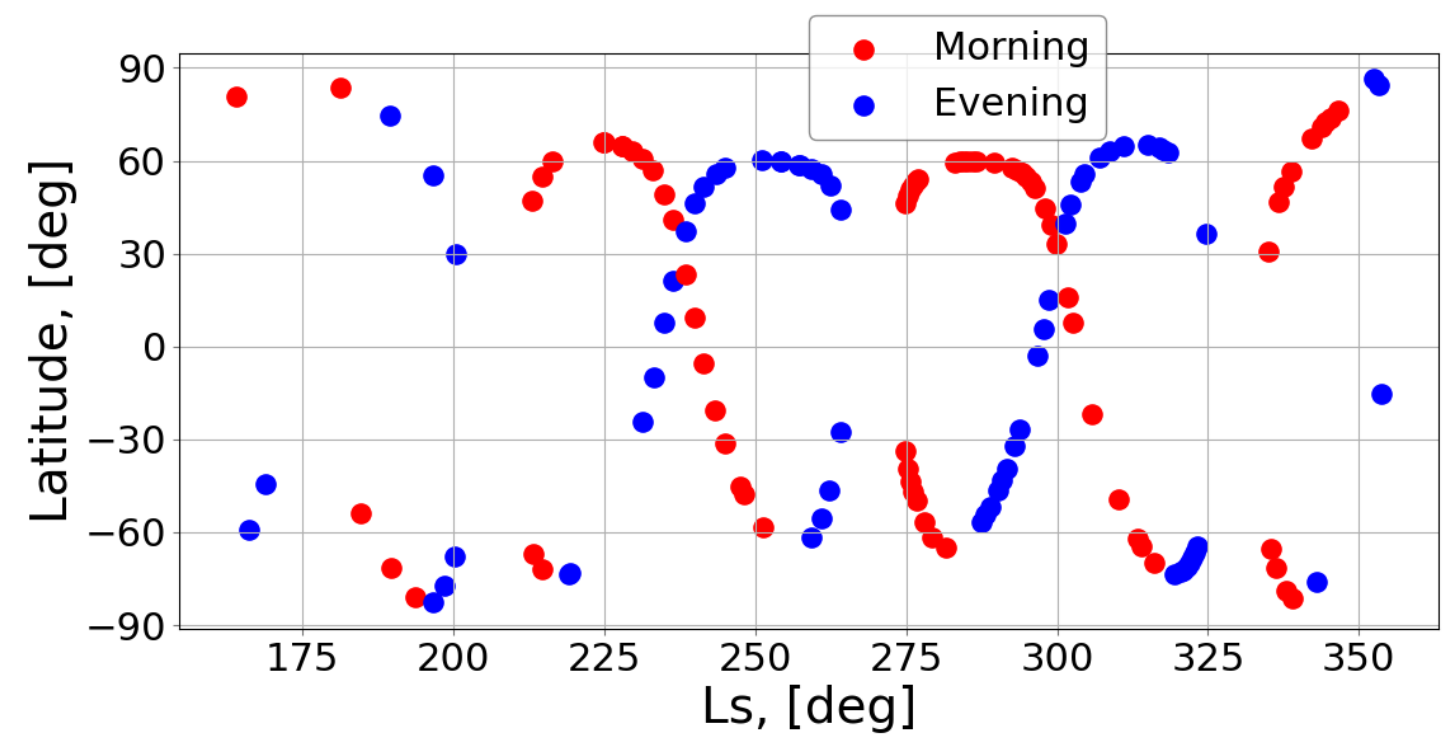

Figure 6. Latitude-solar longitude $\left(L_{s}\right)$ distribution of the ACS MIR occultation profiles used in this study. Morning and evening measurements are shown in red and blue, correspondingly.

A significant portion of observations were made during the global dust storm of MY34, which started between $L_{s}=185^{\circ}$ and $190^{\circ}$, attained its maximum around $L_{s}=220^{\circ}$, and gradually decreased until $L_{s} \approx 290^{\circ}$. A regional storm occurred at the end of MY34 between approximately $L_{s}=325^{\circ}$ and $345^{\circ}$. Figure 7 presents latitude-altitude distribution of the derived GW parameters averaged over the entire period of observations depicted in Figure 6. It shows that the mean amplitude of GW-induced temperature fluctuations $\left(\left|T^{\prime}\right|\right.$, Figure 7a) grows with height reaching up to $\sim 10 \mathrm{~K}$ near the top of the domain. At higher altitudes (170-220 km), the in situ measurements with Neutral Gas and Ion Mass Spectrometer (NGIMS) on board MAVEN revealed even larger GW magnitudes over the same time (Leelavathi et al., 2020; Yiğit et al., 2021). The latitudinal 

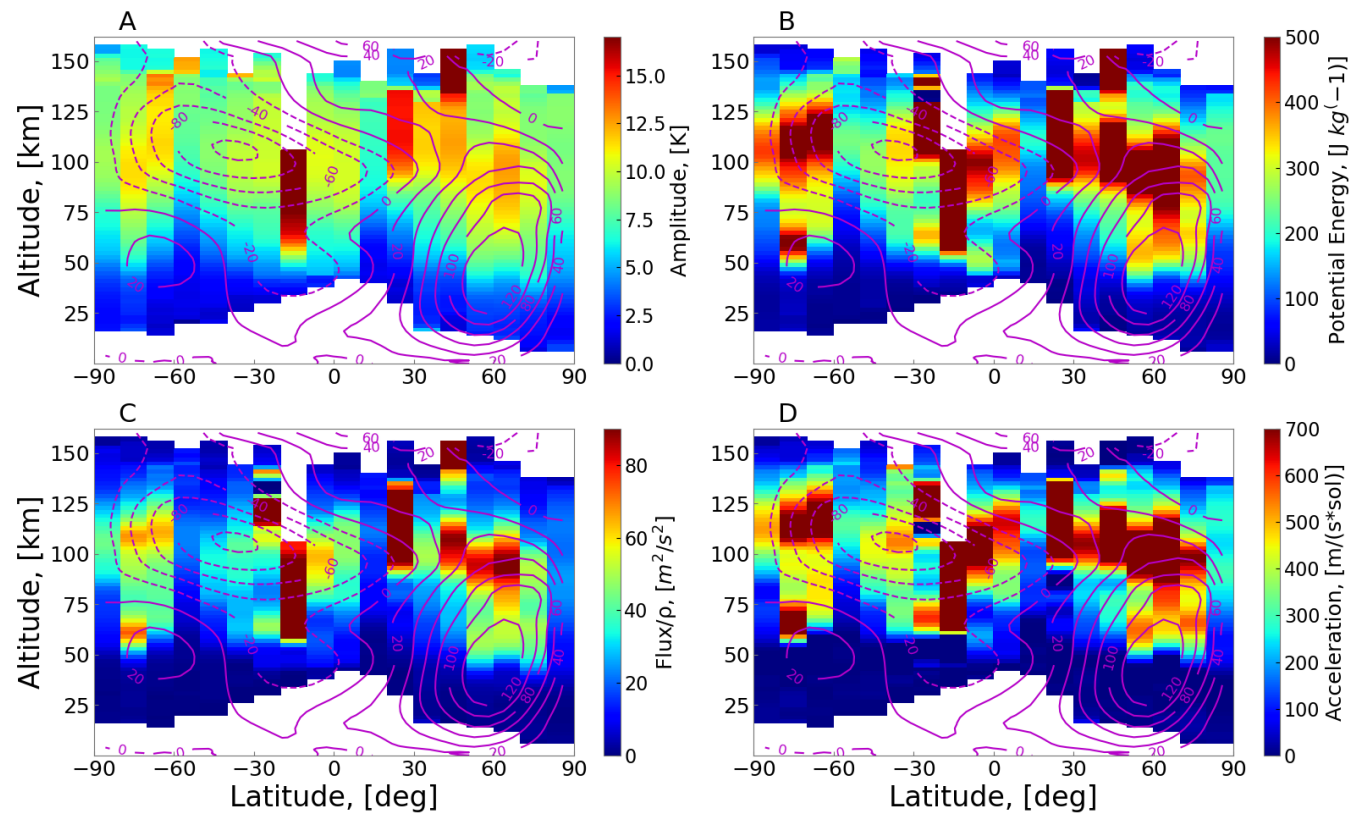

Figure 7. Latitude-altitude cross-sections of the retrieved GW a) amplitudes (in K), b) potential energy (per unit mass), c) vertical fluxes of absolute horizontal momentum (per unit mass) and d) associated momentum forcing ( $\mathrm{GW}$ drag). The size of the employed latitudinal bins is $10^{\circ}$. Contour lines present the zonal wind (in $\mathrm{m} \mathrm{s}^{-1}$ ) simulated with the MAOAM MGCM for MY34 (https://mars.mipt.ru/data.php) and averaged over the same as in Figure 7 period of observations.

structure of the GW activity in the mesosphere and lower thermosphere is not uniform. For comparison, we overplotted the zonal wind simulated with the Max Planck Institute (MAOAM) MGCM https://mars.mipt.ru/ for MY34 and averaged over the same interval of $L_{s}$ as in the observations. The wind distribution varied during this time from the equinoctial to solstitial and back to the equinoctial types. The result reflects the largest contribution of the prograde and retrograde jets during the perihelion solstice. It is seen that the regions with large wave amplitudes encircle the upper edges of two midlatitude jets. This is the result of intensive filtering of individual harmonics by strong background winds. For the wave potential energy, which is a quadratic function of wave amplitudes, this pattern is even more obvious (Figure $7 \mathrm{~b}$ ).

Figure 7c shows that GW momentum fluxes reach local maxima near the mesopause (100-125 km) giving evidence of very intensive wave breakdown/dissipation in this region. The peaks of the associated momentum deposition approximately coincide (Figure 7d). They too wrap around the edges of the jets in the middle atmosphere. It is noteworthy that such distribution of the GW drag is very similar to that predicted by a Martian GCM (Medvedev et al., 2011, Figures 3 and 7) for the solstice and equinox, respectively, and represents the first (to the best of our knowledge) observational validation of the model predictions. The magnitudes of the GW drag, although defined up to the constant $k_{h}$, agree with the simulations (using a similar $k_{h}$ ) as well. 


\subsection{Amplitude Dependence on Mean Temperature and Brunt-Väisälä Frequency}

In situ measurements with NGIMS on board MAVEN showed a clear anti-correlation between relative density fluctuations in the upper thermosphere and the ambient temperature (Yiğit et al., 2015; England et al., 2017; Terada et al., 2017; Vals et al., 2019). It was linked to convective instability as a dominant mechanism that limits growth of GW amplitudes with height (wave saturation). The arguments were based on the relation for a single harmonic (e.g., Fritts et al., 1988, Eq. 6)

$$
\frac{\left|T^{\prime}\right|}{\bar{T}}=\frac{\left|u^{\prime}\right|}{|c-\bar{u}|} \frac{N^{2}}{m g},
$$

where $\left|u^{\prime}\right|$ is the amplitude of fluctuations of horizontal velocity in the wave, $c$ is its horizontal phase velocity and $\bar{u}$ is the background wind. When $\left|u^{\prime}\right|$ approaches $|c-\bar{u}|$, increasing dissipation limits $\left|u^{\prime}\right|$ thus that the ratio $\left|u^{\prime}\right| /|c-\bar{u}|$ becomes constant. The linear convective instability threshold demands a unit ratio, however observations suggested a ratio of 0.7 (Fritts et al., 1988, Eq. 2), and the theoretical consideration of the nonlinear diffusion mechanism yielded $1 / \sqrt{2} \approx 0.707$ (Medvedev \& Klaassen, 2000, Sect. 7). Regardless of the precise number, (7) establishes proportionality between the amplitude of relative temperature/density perturbations and squared mean Brunt-Väisälä frequency under the saturation condition. Near the exobase, where the majority of NGIMS/MAVEN observations were taken, the vertical gradient $d \bar{T} / d z$ is small and can be neglected in (4), thus giving the inverse proportionality of relative perturbation amplitudes and $\bar{T}$.

ACS/TGO occultation data cover altitudes below the exobase, where $d \bar{T} / d z$ can no longer be neglected. Therefore, we plotted in Figure 8a the amplitudes of relative temperature perturbations for all orbits as functions of $N^{2}$. It is seen that red and blue dots corresponding to morning and evening measurements show no clear dependence on $N^{2}$ at all altitudes. To explore this further, we over-plotted the linear regression of the form $\left|T^{\prime}\right| / \bar{T}=\alpha N^{2}+\beta$ and put the values of $\alpha$ and $\beta$ in the legend. The coefficients $\alpha$ are far less than those expected from (7), i.e., several tens or hundreds, depending on the characteristic vertical wavenumber $m$. The distinction between morning and evening amplitudes is also insignificant, except above $100 \mathrm{~km}$, where morning values are slightly larger.

Figure $8 \mathrm{~b}$ presents the dependencies of amplitudes of relative temperature disturbances as functions of the mean temperature. They are nearly uniform. Although regression coefficients show a weak negative trends at all altitudes, their magnitudes are much smaller than to those observed previously (of the order of 0.5 to 1 ) near the exobase (Yiğit et al., 2015; England et al., 2017; Terada et al., 2017; Vals et al., 2019). A similar lack of correlation between GW amplitudes and atmospheric temperature was found from TGO aerobraking measurements at altitudes between 100 and $130 \mathrm{~km}$ (Jesch et al., 2019, Figure 12). The atmospheric drag data were collected between $L_{s}=332^{\circ}$ of $\mathrm{MY} 33$ and $L_{s}=132^{\circ}$ of MY34. The ACS observations after the aerobraking cover the dusty second half of MY34. Thus, the absence of correlation between GW amplitudes and the background temperature in the lower thermosphere appear to be independent of the season and dust conditions. In the upper thermosphere, (Leelavathi et al., 2020, Figure 10d) found a positive correlation during the same second half of MY34, instead of a clear negative correlation over the first ("non-dusty") half of the year. Our results in the adjacent region (around $140 \mathrm{~km}$ ) show no visible change, neither strong negative trend previously found in the MAVEN/NGIMS observations, nor indication of a positive trend. This means that convective instability may not be the main mechanism responsible for damping GWs in the thermosphere, at least during dust storms. 

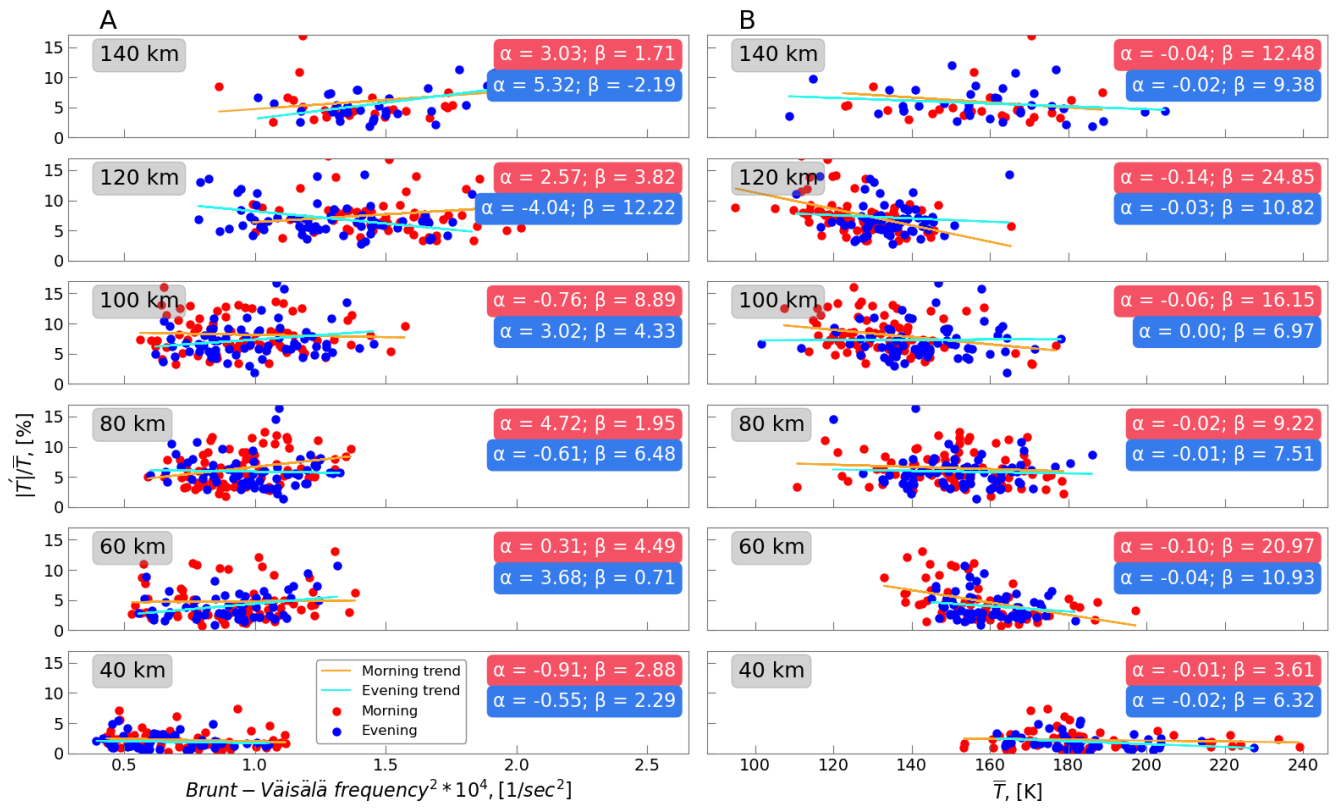

Figure 8. Amplitudes of relative temperature disturbances as functions of the squared BruntVäisälä frequency (a) and mean temperatue (b) at different heights. Red and blue dots are for the morning and evening measurements, correspondingly. Linear regressions of the form a) $\left|T^{\prime}\right| / \bar{T}=\alpha N^{2}+\beta$ and b) $\left|T^{\prime}\right| / \bar{T}=\alpha \bar{T}+\beta$ are shown with thin solid lines, and the values of the respective coefficients are given in the legends.

\section{Summary and Conclusions}

We have presented the results of gravity wave (GW) retrievals obtained from the Atmospheric Chemistry Suite instrument on board the ExoMars Trace Gas Orbiter (ACS/TGO), which observed solar occultation spectra. GW disturbances are derived from the vertical temperature profiles retrieved from one of the three instrument channels - the midinfrared ACS/MIR. The uniqueness of the data is that they continuously cover a broad range of altitudes from the Martian troposphere to the thermosphere $(20-160 \mathrm{~km})$ and have a relatively high $(0.5$ to $2.5 \mathrm{~km})$ vertical resolution.

Several techniques of separating GW components from the background temperature have been studied. The sliding-window least square polynomial fitting method have demonstrated to be the most robust and effective. The procedure was applied to 144 measurements collected over the second half of MY34 to derive vertical profiles of GW disturbances as well as further wave characteristics: amplitude, wave potential energy, absolute vertical flux of horizontal momentum and absolute momentum forcing produced by breaking/dissipating GWs ("GW drag"). The main results are listed below.

1. Amplitudes of GW-induced temperature fluctuations, generally, grow with height, while breaking/saturation processes often limit the wave amplitude growth at higher altitudes. Based on a half-year average, wave amplitudes are around 8-14 K near the mesopause, and often exceed these values in individual profiles. 
2. The mesopause (100-120 km) is the region of the strongest GW breaking/dissipation, which is evidenced by a local maximum of momentum fluxes and their vertical divergence, i.e., GW drag. Similarly, a large GW drag of hundreds of $\mathrm{m} \mathrm{s}^{-1} \mathrm{sol}^{-1}$ in the mesopause region has been demonstrated by MGCMs (e.g., Yiğit et al., 2018).

3. The spatial (altitude-latitude) distribution of the wave drag also agrees well with modeling results (e.g., Medvedev et al., 2011). This is the first direct observational validation of model predictions.

4. We did not find positive correlation between amplitudes of relative temperature perturbations and the Brunt-Väisälä frequency at all heights. This correlation is a more general formulation of the anti-correlation found near the exobase (Yiğit et al., 2015; England et al., 2017; Terada et al., 2017; Vals et al., 2019) that accounts for vertically varying mean temperature.

The presented GW activity retrievals extending from the middle troposphere to the thermosphere, as derived from the ExoMars data, highlight the role of atmospheric gravity waves as a whole atmosphere phenomenon on Mars. Mars' thin and windy atmosphere favors strong gravity wave generation, thus an accurate characterization of gravity waves is absolutely essential for a better understanding of the Martian climate (Yiğit \& Medvedev, 2019).

\section{Acknowledgments}

ExoMars is the space mission of ESA and Roscosmos. The ACS experiment is led by IKI Space Research Institute in Moscow. Science operations of ACS are funded by Roscosmos and ESA. The analyses of temperature profiles and wave structures at IKI are funded by the grant \#20-42-09035 of the Russian Science Foundation. The ACS data are available from ESA's Planetary Science Archive at https://archives.esac.esa.int/psa/ \#! Table $\backslash \% 20$ View/ACS=instrument. The data for latitude-altitude cross-sections of GW characteristics such as amplitudes, potential energy, fluxes, accelerations and zonal wind are available at the Mendeley database (Starichenko, 2021).

\section{References}

Alday, J., Wilson, C. F., Irwin, P. G. J., Olsen, K. S., Baggio, L., Montmessin, F., ... Shakun, A. (2019). Oxygen isotopic ratios in Martian water vapour observed by ACS MIR on board the ExoMars Trace Gas Orbiter. Astronomy \& Astrophysics, 630. doi: 10.1051/0004-6361/201936234

Alexander, M. J. (1998). Interpretations of observed climatological patterns in stratospheric gravity wave variance. Journal of Geophysical Research, 103(D8), 8627-8640. Retrieved from https://agupubs.onlinelibrary.wiley.com/ doi/pdf/10.1029/97JD03325 doi: 10.1029/97JD03325

Ando, H., Imamura, T., \& Tsuda, T. (2012). Vertical wavenumber spectra of gravity waves in the Martian atmosphere obtained from Mars Global Surveyor radio occultation data. Journal of the Atmospheric Sciences, 69, 2906-2912. doi: 10.1175/JAS-D-11-0339.1

Creasey, J. E., Forbes, J. M., \& Hinson, D. P. (2006b). Global and seasonal distribution of gravity wave activity in Mars' lower atmosphere derived from MGS radio occultation data. Geophysical Research Letters, $33 . \quad$ doi: 10.1029/2005GL024037

Creasey, J. E., Forbes, J. M., \& Keating, G. M. (2006a). Density variability at scales typical of gravity waves observed in Mars' thermosphere by the MGS accelerometer. Geophysical Research Letters, 33(L22814). Retrieved from https://agupubs.onlinelibrary.wiley.com/doi/full/10.1029/ 2006GL027583 doi: 10.1029/2006GL027583

Ehard, B., Kaifler, B., Kaifler, N., \& Rapp, M. (2015). Evaluation of methods 
for gravity wave extraction from middle-atmospheric lidar temperature measurements. Atmospheric Measurement Techniques, 8(11), 4645-4655. Retrieved from https://amt . copernicus.org/articles/8/4645/2015/ doi: 10.5194/amt-8-4645-2015

England, S. L., Liu., G., Yiğit, E., Mahaffy, P. R., Elrod, M., Benna, M., ... Jakosky, B. (2017). MAVEN NGIMS observations of atmospheric gravity waves in the Martian thermosphere. Journal of Geophysical Research: Space Physics, 2310-2335. doi: 10.1002/2016JA023475

Ern, M., Preusse, P., Alexander, M. J., \& Warner, C. D. (2004). Absolute values of gravity wave momentum flux derived from satellite data. Journal of Geophysical Research: Atmospheres, 109. doi: 10.1029/2004JD004752

Fedorova, A. A., Montmessin, F., Korablev, O., Luginin, M., Trokhimovskiy, A., Belyaev, D. A., ... Wilson, C. F. (2020). Stormy water on Mars: The distribution and saturation of atmospheric water during the dusty season. Science, 367, 297-300. Retrieved from https://science.sciencemag.org/content/ sci/367/6475/297.full.pdf doi: 10.1126/science.aay 9522

Forget, F., Montmessin, F., Bertaux, J.-L., González-Galindo, F., Lebonnois, E., Sébastien Quémerais, Reberac, A., ... López-Valverde, M. A. (2009). Density and temperatures of the upper Martian atmosphere measured by stellar occultations with Mars Express SPICAM. Journal of Geophysical Research, 114(E01004). Retrieved from https://doi.org/10.1029/2008JE003086 doi: 10.1029/2008JE003086

Fritts, D. C., \& Alexander, J. M. (2003). Gravity wave dynamics and effects in the middle atmosphere. Reviews of Geophysics, 41(1), 1003. Retrieved from https://agupubs.onlinelibrary.wiley.com/doi/full/10.1029/ 2001RG000106 doi: 10.1029/2001RG000106

Fritts, D. C., Tsuda, T., Kato, S., Sato, T., \& Fukao, S. (1988). Observational Evidence of a Saturated Gravity Wave Spectrum in the Troposphere and Lower Stratosphere. Journal of the Atmospheric Sciences, 45 (12), 17411759. Retrieved from https://journals.ametsoc.org/view/journals/ atsc/45/12/1520-0469_1988_045_1741_oeoasg_2_0_co_2.xml doi: 10.1175/1520-0469(1988)045〈1741:OEOASG $>2.0$. CO;2

Fritts, D. C., Wang, L., \& Tolson, R. H. (2006). Mean and gravity wave structures and variability in the Mars upper atmosphere inferred from Mars Global Surveyor and Mars Odyssey aerobraking densities. Journal of Geophysical Research, 111(A12304). Retrieved from https://agupubs.onlinelibrary .wiley.com/doi/10.1029/2006JA011897 doi: 10.1029/2006JA011897

Gamache, R. R., Farese, M., \& Renaud, C. L. (2016). A spectral line list for water isotopologues in the 1100-4100 cm-1 region for application to CO2-rich planetary atmospheres. Journal of Molecular Spectroscopy, 326, 144-150. Retrieved from http://www.sciencedirect.com/science/article/pii/ S0022285215001770 (New Visions of Spectroscopic Databases, Volume I) doi: https://doi.org/10.1016/j.jms.2015.09.001

Gordon, I., Rothman, L., Hill, C., Kochanov, R., Tan, Y., Bernath, P., .. B Boudon, V. (2017). The HITRAN2016 molecular spectroscopic database. Journal of Quantitative Spectroscopy and Radiative Transfer, 203, 3-69. Retrieved from https://www.sciencedirect.com/science/article/pii/ S0022407317301073 doi: 10.1016/j.jqsrt.2017.06.038

Gröller, H., Montmessin, F., Yelle, R. V., Lefèvre, F., Forget, F., Schneider, N. M., \& et al. (2018). MAVEN/IUVS stellar occultation measurements of Mars atmospheric structure and composition. Journal of Geophysical Research: Planets, 123, 1449-1483. Retrieved from https://doi.org/10.1029/ 2017JE005466 doi: 10.1029/2017JE005466

Heavens, N. G., Kass, D. M., Kleinböhl, A., \& Schofield, J. T. (2020). A multiannual record of gravity wave activity in Mars's lower atmosphere from 
on-planet observations by the Mars Climate Sounder. Icarus, 341, 113630. doi: $10.1016 /$ j.icarus.2020.113630

Hinson, D. P., Simpson, R. A., Twicken, J. D., Tyler, G. L., \& Flasar, F. M.

(1999). Initial results from radio occultation measurements with Mars Global Surveyor. Journal of Geophysical Research: Planets, 104(E11), 2699727012. Retrieved from https://doi.org/10.1029/1999JE001069 doi: 10.1029/1999JE001069

Irwin, P., Teanby, N., de Kok, R., Fletcher, L., Howett, C., Tsang, C., ... Parrish, P. (2008). The NEMESIS planetary atmosphere radiative transfer and retrieval tool. Journal of Quantitative Spectroscopy and Radiative Transfer, 109(6), 1136-1150. Retrieved from https://www.sciencedirect.com/science/ article/pii/S0022407307003378 (Spectroscopy and Radiative Transfer in Planetary Atmospheres) doi: 10.1016/j.jqsrt.2007.11.006

Jesch, D., Medvedev, A. S., Castellini, F., Yiğit, E., \& Hartogh, P. (2019). Density Fluctuations in the Lower Thermosphere of Mars Retrieved From the ExoMars Trace Gas Orbiter (TGO) aerobraking. Atmosphere, 10(10). Retrieved from https://www.mdpi.com/2073-4433/10/10/620 doi: 10.3390/atmos10100620

John, S. R., \& Kumar, K. K. (2013). A discussion on the methods of extracting gravity wave perturbations from space-based measurements. Geophysical Research Letters, 40(10), 2406-2410. Retrieved from https:// agupubs.onlinelibrary.wiley.com/doi/abs/10.1002/grl.50451 doi: $10.1002 /$ grl.50451

Keating, G. M., Bougher, S. W., Zurek, R. W., Tolson, R. H., Cancro, G. J., Noll, S. N., .. Babicke, J. M. (1998). The Structure of the Upper Atmosphere of Mars: In Situ Accelerometer Measurements from Mars Global Surveyor. Science, 279(5357), 1672-1676. Retrieved from https://science.sciencemag .org/content/279/5357/1672 doi: 10.1126/science.279.5357.1672

Korablev, O., Montmessin, F., Trokhimovskiy, A., Fedorova, A., Shakun, A., Grigoriev, A., \& et al. (2018). The Atmospheric Chemistry Suite (ACS) of three spectrometers for the ExoMars 2016 Trace Gas Orbiter. Space Science Reviews, 214(7). Retrieved from https://link.springer.com/article/ 10.1007/s11214-017-0437-6 doi: 10.1007/s11214-017-0437-6

Leelavathi, V., Venkateswara Rao, N., \& Rao, S. V. B. (2020). Interannual Variability of Atmospheric Gravity Waves in the Martian thermosphere: Effects of the 2018 Planet-encircling Dust Event. Journal of Geophysical Research: Planets, 125 (12), e2020JE006649. doi: 10.1029/2020JE006649

Marquardt, D. W. (1963). An Algorithm for Least-Squares Estimation of Nonlinear Parameters. Journal of the Society for Industrial and Applied Mathematics, 11(2), 431-441. Retrieved from https://doi.org/10.1137/0111030 doi: 10 $.1137 / 0111030$

Medvedev, A. S., \& Klaassen, G. P. (2000). Parameterization of gravity wave momentum deposition based on nonlinear wave interactions: Basic formulation and sensitivity tests. JASTP, 62, 1015-1033. doi: 10.1016/ S1364-6826(00)00067-5

Medvedev, A. S., Nakagawa, H., Mockel, C., Yiğit, E., Kuroda, T., Hartogh, P., ... Jakosky, B. M. (2016). Comparison of the Martian thermospheric density and temperature from IUVS/MAVEN data and general circulation modeling. Geophysical Research Letters, 43(7), 3095-3104. doi: 10.1002/2016GL068388

Medvedev, A. S., \& Yiğit, E. (2019). Gravity Waves in Planetary Atmospheres: Their Effects and Parameterization in Global Circulation Models. Atmosphere, 10 (9). doi: 10.3390/atmos10090531

Medvedev, A. S., Yiğit, E., Hartogh, P., \& Becker, E. (2011). Influence of gravity waves on the Martian atmosphere: General circulation modeling. Journal of Geophysical Research, 116(E10004). Retrieved from https://doi.org/10 .1029/2011JE003848 doi: 10.1029/2011JE003848 
Millour, E., Forget, F., Spiga, A., Vals, M., Zakharov, V., Montabone, L., \& et al. (2018). The mars climate database (version 5.3). Paper presented at the Mars Science Workshop "From Mars Express to ExoMars", held 27-28 February 2018 at ESAC, Madrid, Spain, id.68.. Retrieved from https://www . cosmos.esa.int/web/mars-science-workshop-2018

Nakagawa, H., Terada, N., Jain, S. K., Schneider, N. M., Montmessin, F., Yelle, R. V., ... Jakosky, B. M. (2020). Vertical Propagation of Wave Perturbations in the Middle Atmosphere on Mars by MAVEN/IUVS. Journal of Geophysical Research: Planets, 125(9), e2020JE006481. Retrieved from https:// agupubs . onlinelibrary.wiley.com/doi/abs/10.1029/2020JE006481 (e2020JE006481 2020JE006481) doi: https://doi.org/10.1029/2020JE006481

Sen, P. K. (1968). Estimates of the Regression Coefficient Based on Kendall's Tau. Journal of the American Statistical Association, 63(324), 1379-1389. Retrieved from https://www.jstor.org/stable/2285891 doi: 10.2307/2285891

Siddle, A., Mueller-Wodarg, I., \& Bruinsma, J.-C. M. (2020). Density structures in the Martian lower thermosphere as inferred by Trace Gas Orbiter accelerometer measurements. Icarus. Retrieved from https://www. sciencedirect.com/ science/article/pii/S0019103520304541?vial\%3Dihub doi: 10.1016/ j.icarus.2020.114109

Spiga, A., Teitelbaum, H., \& Zeitlin, V. (2008). Identification of the sources of inertia-gravity waves in the Andes Cordillera region. Annales Geophysicae, 26(9), 2551-2568. Retrieved from https://angeo.copernicus.org/ articles/26/2551/2008/ doi: 10.5194/angeo-26-2551-2008

Starichenko, E. D. (2021). Gravity wave activity in the Martian atmosphere at altitudes 20-160 km from ACS/TGO occultation measurements. Retrieved from https://data.mendeley.com/datasets/gk6sr9f wzp/draft?a=a1907fab -d5b6-4603-8e43-e234884f259a doi: 10.17632/gk6sr9fwzp.1

Terada, N., Leblanc, F., Nakagawa, H., Medvedev, A. S., Yiğit, E., Kuroda, T., ... Jakosky, B. M. (2017). Global distribution and parameter dependences of gravity wave activity in the Martian upper thermosphere derived from MAVEN/NGIMS observations. Journal of Geophysical Research: Space Physics, 122(2), 2374-2397. Retrieved from https:// agupubs.onlinelibrary.wiley.com/doi/abs/10.1002/2016JA023476 doi: 10.1002/2016JA023476

Theil, H. (1950). A rank-invariant method of linear and polynomial regression analysis. Proceedings van de Koninklijke Nederlandse Akademie van Wetenschappen, 53, 386-392, 521-525, 1397-1412. Retrieved from https://ir.cwi.nl/pub/ 18445

Tikhonov, A. N., \& Arsenin, V. Y. (1977). Solutions of Ill-Posed Problems. Washington, D. C.: V. H. Winston.

Tolson, R., Bemis, E., Hough, S., Zaleski, K., Keating, G., Shidner, J., ... Thomas, P. (2008). Atmospheric modeling using accelerometer data during Mars Reconnaissance Orbiter aerobraking operations. Journal of Spacecraft and Rockets, 45(3). Retrieved from https://arc.aiaa.org/doi/abs/10.2514/1.34301 doi: $10.2514 / 1.34301$

Tolson, R. H., Keating, G. M., George, B. E., Escalera, P. E., \& Werner, M. R. (2005). Application of Accelerometer Data to Mars Odyssey Aerobraking and Atmospheric Modeling. Journal of Spacecraft and Rockets, 45(3). Retrieved from https://arc.aiaa.org/doi/10.2514/1.15173 doi: 10.2514/1.15173

Vals, M., Spiga, A., Forget, F., Millour, E., Montabone, L., \& Lott, F. (2019). Study of gravity waves distribution and propagation in the thermosphere of Mars based on MGS, ODY, MRO and MAVEN density measurements. Planetary and Space Science, 178(104708). Retrieved from https://doi.org/10.1016/ j.pss.2019.104708 doi: $10.1016 /$ j.pss.2019.104708

Whiteway, J., \& Carswell, A. (1995). Lidar observations of gravity wave activity 
in the upper stratosphere over Toronto. Journal of Geophysical Research, 100(D7), 14113-14124. Retrieved from https://agupubs.onlinelibrary .wiley.com/doi/abs/10.1029/95JD00511 doi: 10.1029/95JD00511

Withers, P. (2006). Mars Global Surveyor and Mars odyssey accelerometer observations of the Martian upper atmosphere during aerobraking. Geophysical Research Letters, 33. doi: 10.1029/2005GL024447

Wright, C. J. (2012, May). A one-year seasonal analysis of Martian gravity waves using MCS data. Icarus, 219(1), 274-282. Retrieved 2020-06-27, from https://linkinghub.elsevier.com/retrieve/pii/S0019103512000899 doi: $10.1016 /$ j.icarus.2012.03.004

Yiğit, E., England, S. L., Liu, G., Medvedev, A. S., Mahaffy, P. R., Kuroda, T., \& Jakosky, B. M. (2015). High-altitude gravity waves in the Martian thermosphere observed by MAVEN/NGIMS and modeled by a gravity wave scheme. Geophysical Research Letters, 42(21), 8993-9000. Retrieved from https:// agupubs.onlinelibrary.wiley.com/doi/abs/10.1002/2015GL065307 doi: 10.1002/2015GL065307

Yiğit, E., \& Medvedev, A. S. (2015). Internal wave coupling processes in Earth's atmosphere. Advances in Space Research, 55(4), 983-1003. Retrieved from https://www.sciencedirect.com/science/article/abs/pii/ S0273117714007236 doi: 10.1016/j.asr.2014.11.020

Yiğit, E., \& Medvedev, A. S. (2019). Obscure waves in planetary atmospheres. Physics Today, 6, 40-46. doi: 10.1063/PT.3.4226

Yiğit, E., Medvedev, A. S., Benna, M., \& Jakosky, B. M. ～(2021). Dust stormenhanced gravity wave activity in the Martian thermosphere observed by MAVEN and implication for atmospheric escape. Geophysical Research Letters. doi: 10.1029/2020GL092095

Yiğit, E., Medvedev, A. S., \& Hartogh, P. (2018). Influence of gravity waves on the climatology of high-altitude Martian carbon dioxide ice clouds. Annales Geophysicae, 36(6), 1631-1646. doi: 10.5194/angeo-36-1631-2018 